\title{
Evolution of water dynamics in the Prussian blue
}

\author{
S. Mitra ${ }^{1}$, V.K. Sharma ${ }^{1}$, N. Thakur ${ }^{1}$, S.M. Yusuf ${ }^{1}$, Fanni Juranyi ${ }^{2}$ and R. Mukhopadhyay ${ }^{1}$ a \\ ${ }^{1}$ Solid State Physics Division, Bhabha Atomic Research Centre, Mumbai 400085, India \\ ${ }^{2}$ Laboratory for Neutron Scattering, Paul Scherrer Institut, Villigen, Switzerland
}

\begin{abstract}
Crystal water plays a crucial role towards the multifunctional properties of Prussian blue and its analogue (PBA) compounds. We have investigated the dynamics of crystal water in Prussian blue (PB), $\mathrm{Fe}(\mathrm{III})_{4}\left[\mathrm{Fe}(\mathrm{II})(\mathrm{CN})_{6}\right]_{3} .14 \mathrm{H}_{2} \mathrm{O}$ using Quasielastic Neutron Scattering (QENS). In PB, water molecules exist in the spherical cavity, created due to the vacant sites of $\mathrm{Fe}(\mathrm{CN})_{6}$ units, and also at interstitial sites. QENS data showed that dynamics evolves with increase in temperature. The observed dynamics has been correlated quantitatively with the different water molecules that exist at different sites of the lattice. It is found that only the non-coordinated water molecules contribute to the dynamics at low temperature, and others start contributing progressively with increasing temperature. A detailed data analysis showed that the water molecules undergo a localized translational diffusion. The estimated spatial domain of dynamics is found to be compatible with the geometry of the structure.
\end{abstract}

\section{Introduction}

Prussian blue analogues (PBA) are important compounds due to their various interesting multifunctional properties upon application of external stimuli, such as temperature, magnetic field, light, pressure and humidity [1,2]. These compounds also fall in the category of hexacyanometalates, a known type of molecular magnetic compounds. Molecular magnets have many useful additional properties over conventional magnets, such as low density, flexiblity, magneto-optical properties, and biocompatibility. PBAs are also considered as potential candidates for hydrogen storage materials. Hexacyanometallates can be represented by the general formula $\mathrm{A}_{x}\left[\mathrm{~B}(\mathrm{CN})_{6}\right]_{y} . \mathrm{H}_{2} \mathrm{O}$, where $A$ and $B$ are $3 \mathrm{~d}$ transition metal ions. These compounds possess a $f c c$ structure in which $A$ and $B$ are surrounded octahedrally by $N$ and $C$ atoms, respectively. When $\mathrm{x} / \mathrm{y}=1$, the first coordinations of $A$ and $B$ are $\mathrm{A}[\mathrm{NC}]_{6}$ and $\mathrm{B}[\mathrm{CN}]_{6}$, respectively. In this case, water molecules occupy the interstitial positions [3]. However, when $\mathrm{x} / \mathrm{y}>1$, some of the $\mathrm{B}[\mathrm{CN}]_{6}$ units are vacant and filled by water molecules. Three structurally distinguishable water molecules, (i) coordinated water molecules which are coordinated at $A$ octahedra at $24 \mathrm{e}(\mathrm{x}, 0,0)$ site, (ii) non-coordinated water molecules connected by hydrogen bonds to the coordinated ones in the spherical cavity of $\left[\mathrm{B}(\mathrm{CN})_{6}\right]$ at $32 \mathrm{f}(\mathrm{x}, \mathrm{x}, \mathrm{x})$ site, and (iii) non-coordinated water at $8 \mathrm{c}$ $(1 / 4,1 / 4,1 / 4)$ site of unit cell octants are found in these compounds. The structures of these compounds have been very well studied using $\mathrm{x}$-ray as well as neutron diffraction techniques $[3,4]$. Since water plays a crucial role towards the multifunctional properties of PBA compounds, it is of interest to study their dynamical behaviour. Earlier, we studied the dynamics of water molecules in the PBA compound $\mathrm{Cu}_{0.75} \mathrm{Mn}_{0.75}\left[\mathrm{Fe}(\mathrm{CN})_{6}\right]$. $7 \mathrm{H}_{2} \mathrm{O} \quad[5,6]$.

${ }^{a}$ Corresponding author: mukhop@barc.gov . in
The present work is based on the $\mathrm{Fe}(\mathrm{III})_{4}\left[[\mathrm{Fe}(\mathrm{II})(\mathrm{CN})]_{6}\right]_{3}$. $14 \mathrm{H}_{2} \mathrm{O}$ compound which is different in respect to the available vacant sites as well as dimension of the cavities.

In the Prussian Blue (PB), $\mathrm{Fe}(\mathrm{III})_{4}\left[\mathrm{Fe}(\mathrm{III})(\mathrm{CN})_{6}\right]_{3}$. $14 \mathrm{H}_{2} \mathrm{O}$, owing to the $4: 3$ (x: y) stoichiometry, the charge neutrality necessitates that $1 / 4$ of the $\mathrm{Fe}(\mathrm{CN})_{6}$ sites are vacant. These vacant sites are filled by the water molecules [4]. All three structurally distinguishable kinds of water molecules as mentioned above are found in PB. Out of 14 water molecules, six water molecules are coordinated to $\mathrm{Fe}(\mathrm{III})$ at the empty nitrogen sites $24 \mathrm{e}(\mathrm{x}, 0,0)$ site, four non-coordinated water molecules at $32 \mathrm{f}(\mathrm{x}, \mathrm{x}, \mathrm{x})$ site inside the spherical cavity, and the remaining four noncoordinated water molecules are found at the $8 \mathrm{c}(1 / 4,1 / 4$, 1/4) site of unit cell octants [4]. Therefore, this compound, $\mathrm{PB}$, provides an ideal structure to study the evolution of dynamics of different kinds of crystal water molecules with temperature. Figure 1 shows the typical spherical cavity formed by the coordinated water molecules in case of PB. The non-coordinated water molecules are omitted for clarity. In this paper, we report the results of QENS studies on dynamics of water in $\mathrm{Fe}(\mathrm{III})_{4}\left[\mathrm{Fe}(\mathrm{II})(\mathrm{CN})_{6}\right]_{3}$. $14 \mathrm{H}_{2} \mathrm{O}$ in the temperature range $260-360 \mathrm{~K}$.

\section{Experimental details}

The polycrystalline sample of $\mathrm{PB}$ was prepared by the precipitation method. $\mathrm{PB}$ was prepared by mixing $0.1 \mathrm{M}$ aqueous solution of $\mathrm{K}_{4} \mathrm{Fe}(\mathrm{CN})_{6}$, to rapidly stirred aqueous solution of $0.1 \mathrm{MFeCl}_{3}$, and the resulting solution was heated up to $325 \mathrm{~K}$. The hot solution was allowed to cool at room temperature, and diluted to double of its initial volume after cooling. The obtained precipitates were filtered, washed many times with distilled water, and finally dried at room temperature. These samples were characterized by $\mathrm{x}$-ray as well as neutron diffraction.

This is an Open Access article distributed under the terms of the Creative Commons Attribution License 4.0, which permits unrestricted use, distribution, and reproduction in any medium, provided the original work is properly cited. 




Figure 1. Unit cell of Prussian blue $\mathrm{Fe}(\mathrm{III})_{4}\left[\mathrm{Fe}(\mathrm{II})(\mathrm{CN})_{6}\right]_{3}$. $14 \mathrm{H}_{2} \mathrm{O}$. The spherical cavity formed by coordinated water molecules in PB is indicated.

QENS experiments were performed on the hydrated as well as the dehydrated PB sample in the temperature range $260-360 \mathrm{~K}$ using the hybrid time of flight spectrometer 'FOCUS' at SINQ, Paul Scherrer Institut, Switzerland. The incident wavelength of $6 \AA$ corresponding to an energy resolution (FWHM) of $\Delta E \sim 45 \mu \mathrm{eV}$ was used for the QENS experiment. The samples were placed in a flat rectangular aluminum can with an internal spacing of $0.2 \mathrm{~mm}$ (which ensured scattering not more than 10\%) such that multiple scattering effects could be neglected. The data from the dehydrated sample were used to estimate the contribution from the sample without water. For dehydration, sample was heated at $420 \mathrm{~K}$ under vacuum $(15 \mathrm{mb})$ for a period of about $10 \mathrm{~h}$. The dehydrated sample was then sealed in a glove box and used for the neutron scattering measurements.

\section{Results and discussions}

Significant quasielastic (QE) broadening is observed for the hydrated $\mathrm{PB}$ sample over the instrument resolution whereas no QE broadening is observed for the dehydrated sample. Therefore, the observed QE broadening corresponds to dynamics of water. This is also corroborated by the vibrational density of states (DOS) as obtained using the following expression [7]

$$
g(E) \cong \frac{E}{Q^{2}}\left[1-\exp \left(-\frac{E}{K_{B} T}\right)\right] S(Q, E)
$$

where $K_{B}$ is Boltzman constant, $T$ is temperature, $E$ is energy transfer and $Q$ is wavevector transfer. The peak around $8 \mathrm{meV}$ present in the hydrated form, corresponding to the intermolecular bending motions of the water molecules $[7,8]$, is found to be absent in the dehydrated sample as shown in Fig. 2. An absence of peak at $8 \mathrm{meV}$ in the dehydrated sample suggests that sample is devoid of water.

QENS data as obtained for the dehydrated sample were subtracted from that of the hydrated samples to extract the contribution from the water alone. Typical subtracted QENS spectra for PB are shown in Fig. 3 at $\mathrm{Q}=$ $1.1 \AA^{-1}$ for different temperatures. It is evident that the



Figure 2. Vibrational DOS in PB in hydrated and dehydrated form at $300 \mathrm{~K}$.

quasielastic broadening is clearly seen even at $260 \mathrm{~K}$. This is mostly because the water molecules inside PB exist in a confined state, and some water molecules are coordinated to Fe octahedra. These confined water molecules show the supercooling effect as also observed in many confined systems. On increase of temperature from $260 \mathrm{~K}$ to $360 \mathrm{~K}$, the $\mathrm{QE}$ broadening is found to increase gradually. To separate the elastic and quasielastic components of the subtracted data, the following general scattering law is used,

$$
\mathrm{S}(Q, \omega)=A(Q) \delta(\omega)+[1-A(Q)] L(\Gamma, \omega)
$$

where the first term is the elastic part and the second term is the quasielastic one. $\mathrm{L}(\Gamma, \omega)$ is a Lorentzian function with a half width at half maxima (HWHM) $\Gamma(\mathrm{Q})$. The observed and fitted data at different temperatures and $Q$ values are shown in Fig. 4. The presence of an elastic contribution, $\mathrm{A}(\mathrm{Q})$ which is nothing but the Elastic Incoherent Structure Factor (EISF) suggests that some water molecules are immobile within the time scale accessible by the spectrometer and/or are undergoing a localised motion. The derived EISF are shown in Fig. 5. The variation of obtained EISF at different temperatures indicates that there is an evolution of dynamics as the temperature is increased.

The observed evolution in dynamics suggests that as the temperature is increased, more number of water molecules contribute to the dynamics. Thus, a generalized scattering law for a system where a fraction of water molecules $\left(P_{x}\right)$ contributes to the dynamics can be written as,

$$
\begin{aligned}
S(Q, \omega)= & \left(1-P_{x}\right) \delta(\omega)+ \\
& P_{x}\left[A_{0}(Q) \delta(\omega)+\left(1-A_{0}(Q)\right) L(\Gamma, \omega)\right](3)
\end{aligned}
$$

where $\mathrm{A}_{0}(\mathrm{Q})$ is the model EISF. Therefore, the total elastic fraction would be, $\left[P_{x} \mathrm{~A}_{0}(\mathrm{Q})+\left(1-P_{x}\right)\right]$. In case of translational diffusion of particles within a sphere of radius $a$, enclosed by impermeable boundary, $\mathrm{A}_{0}(\mathrm{Q})$ can be given as [9]

$$
A_{0}(Q)=\left[\frac{3 j_{1}(Q a)}{Q a}\right]^{2}
$$




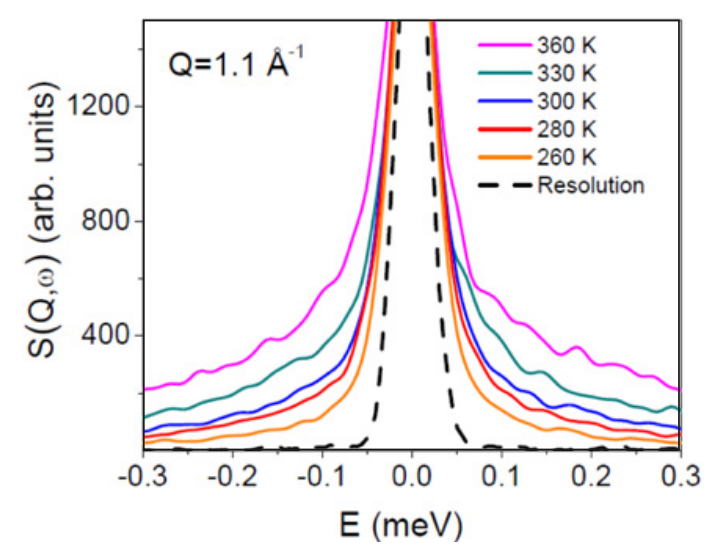

Figure 3. QENS spectra at different temperatures at $Q=$ $1.1 \AA^{-1}$. Contribution from dehydrated sample has been subtracted. The dashed lines show the instrumental resolution. Spectra have been normalized to maximum peak intensity of vanadium.

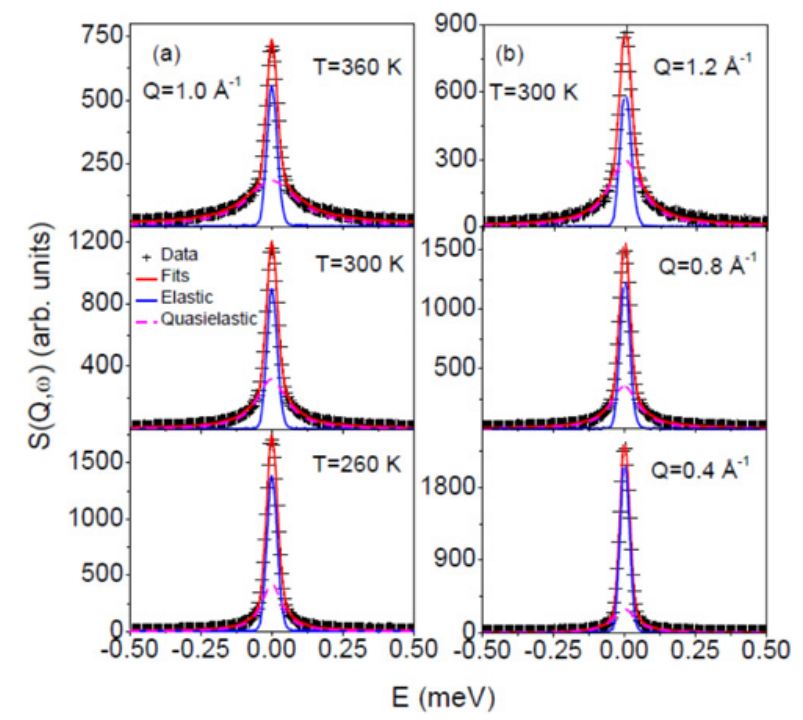

Figure 4. Fitted subtracted QENS spectra (a) at different temperatures at typical $\mathrm{Q}=1.0 \AA^{-1}$ and (b) at different $\mathrm{Q}$ values at $300 \mathrm{~K}$.

In that case, the effective EISF can be written as,

$$
A(Q)=\left[P_{x}\left(\frac{3 j_{1}(Q a)}{Q a}\right)^{2}+\left(1-P_{x}\right)\right] .
$$

Here $j_{1}(Q a)$ is the first order spherical Bessel function. It is found that the above equation could describe the observed EISF very well at all the measured temperatures, shown by the solid lines in Fig. 5. The mobile fraction of the water molecules, $P_{x}$, and the radius of the spherical cavity within which the molecules undergo localized dynamics, $a$, are the parameters determined by the least squares fit of the experimentally obtained EISF. The derived values of $P_{x}$ and $a$ for water are given in Table 1 . The values suggest that as the temperature increases more water molecules participate in the dynamics. However, the obtained value of the radius of the spherical volume $(a)$ is found to be very close to the size of the cavity (radius $\sim 2.8 \AA$ ) formed in $\mathrm{PB}$ due to the vacant sites of $\mathrm{Fe}(\mathrm{CN})_{6}$ [4]. It is

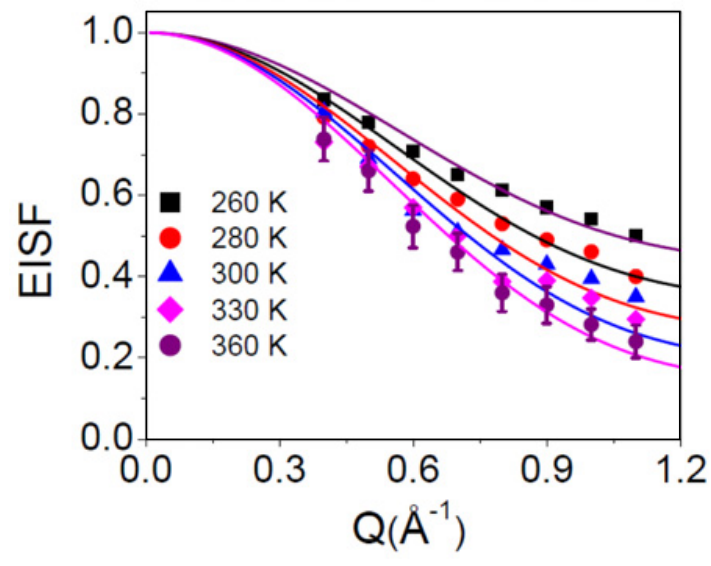

Figure 5. Variation of EISF as obtained from QENS data at different temperatures. Solid lines show the fits using a localized translational diffusion model [Eq. (5)].

Table 1. Fraction of water molecules taking part in the dynamics $\left(P_{x}\right)$, localization radius of sphere $(a)$ and diffusion coefficient $(D)$ at different temperatures.

\begin{tabular}{|c|c|c|c|}
\hline $\mathrm{T}(\mathrm{K})$ & $\begin{array}{c}\text { Fraction of } \\
\text { mobile water }\left(P_{x}\right)\end{array}$ & $a(\AA)$ & $D\left(\times 10^{-5} \mathrm{~cm}^{-2} / \mathrm{s}\right)$ \\
\hline 260 & 0.56 & 2.90 & 0.97 \\
\hline 280 & 0.65 & 2.94 & 1.24 \\
\hline 300 & 0.73 & 2.96 & 1.52 \\
\hline 330 & 0.80 & 2.95 & 1.85 \\
\hline 360 & 0.85 & 3.0 & 2.27 \\
\hline
\end{tabular}

also evident that the fraction of mobile water molecules increases with the increasing temperature. However, the size of spherical domain remains more or less constant with the varying temperature. The most likely picture, emerging from the data analysis is that there exist three kinds of water molecules in the system. The first kind is the coordinated water molecules located at the $24 \mathrm{e}$ site (the spherical cavity formed by vacant $\mathrm{Fe}(\mathrm{CN})_{6}$ units), which are coordinated with $\mathrm{Fe}$ and do not participate in the dynamics at low temperatures. The second kind is the non-coordinated water molecules residing at the interstitial sites $(8 \mathrm{c})$. The third kind of water molecules is non-coordinated ones (at $32 \mathrm{f}$ sites), lie within the spherical cavity. At low temperatures, if only the noncoordinated water molecules i.e., water molecules at $8 \mathrm{c}$ and $32 \mathrm{f}$ sites, are dynamic, (i.e. eight out of a total of fourteen molecules in the formula unit), one can expect the value of $P_{x}$ to be $\sim 57 \%$. Interestingly, the observed mobile fraction as extracted from the QENS data was found to be $56 \%$ at $260 \mathrm{~K}$. Therefore, it can be assumed that at low temperature, the coordinated water molecules are immobile. However, as the temperature is increased, the coordinated water molecules gain enough thermal energy to break the coordination (bonding from $\mathrm{Fe}$ ), and participate in the dynamics progressively.

The variations of the HWHM of quasielastic (QE) component $(\Gamma)$ as a function of $Q$ at different temperatures are shown in Fig. 6. It is clear that the QE width does not go to zero at low $\mathrm{Q}$ value which is a characteristic 


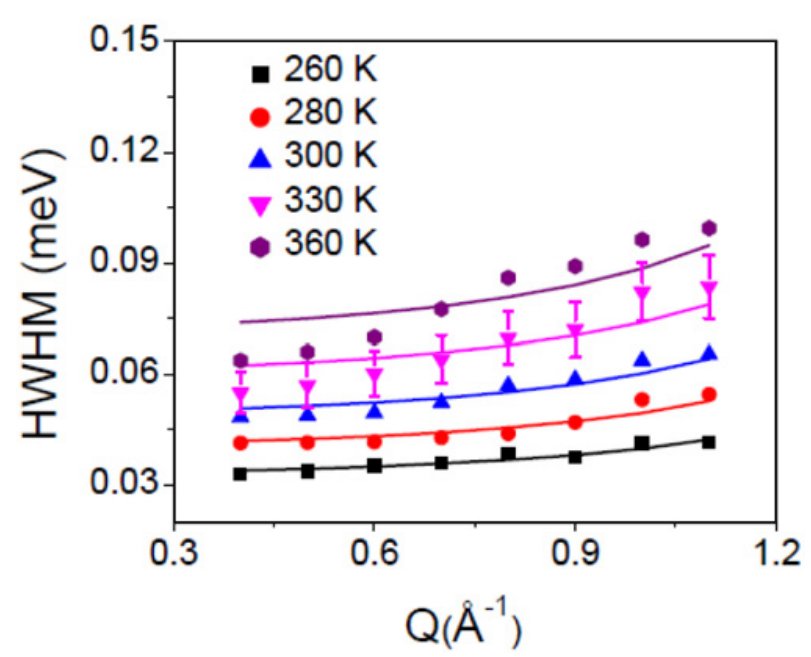

Figure 6. Variation of half width at half maxima (HWHM) of a Lorentzian representing water dynamics with Q. Solid lines are the fit with a model based on localized translational diffusion as described in Eq. (6).

behaviour of localised dynamics. Therefore, the model scattering function for the water molecules performing localised translational diffusion inside a spherical cavity can be given as [9]

$$
\begin{aligned}
S(Q, \omega) & =\left(1-P_{x}\right) \delta(\omega)+ \\
P_{x} & {\left[\begin{array}{l}
A_{0}^{0}(Q a) \delta(\omega)+ \\
\left.\frac{1}{\pi} \sum_{\{l, n\} \neq\{0,0\}}(2 l+1) A_{n}^{l}(Q a) \frac{\left(x_{n}^{l}\right)^{2} D / a^{2}}{\left[\left(x_{n}^{l}\right)^{2} D / a^{2}\right]^{2}+\omega^{2}}\right] .
\end{array}\right.}
\end{aligned}
$$

Here, the first term represents the elastic contribution due to immobile water molecules. Second term represents the scattering function for localised translational diffusion weighted by the fraction of mobile water molecules. Here, $D$ is the diffusion coefficient, and $x_{n}^{l}=a \sqrt{\lambda_{n}^{l}}$ are dimensionless numbers, whose first 99 values are tabulated in Ref. 9. Since no analytical expression exists for the HWHM of the quasielastic part unlike that in case of EISF, the HWHM can be calculated numerically (using Eq. (6)) for given values of $a$ and $D$. The least-squares fitting method is used to describe the observed QE width with $D$ as parameter, while the values of $a$ are already known from the fit of the EISF. Solid lines in Fig. 6 show the fits of the QE widths as obtained assuming the localised translational diffusion model. The derived values of diffusion constants are given in Table 1. At room temperature, diffusion constant for water in PB is found to be $1.36 \times 10^{-5} \mathrm{~cm}^{2} / \mathrm{s}$ which is much hindered as compared to that for bulk water $\left(\sim 2.5 \times 10^{-5} \mathrm{~cm}^{2} / \mathrm{s}\right)$. The diffusion coefficient of water is found to increase with increasing temperature. Activation energy of $\sim 1.6 \mathrm{Kcal} / \mathrm{mol}$ is estimated from the temperature dependence of the diffusion coefficient.

\section{Conclusions}

Dynamics of crystal water in the Prussian blue, $\mathrm{Fe}(\mathrm{III})_{4}\left[\mathrm{Fe}(\mathrm{II})(\mathrm{CN})_{6}\right]_{3} .14 \mathrm{H}_{2} \mathrm{O}$ molecular magnet has been investigated using the quasielastic neutron scattering technique. Contributions from the water molecules were extracted by subtracting the data from the corresponding dehydrated samples. It is found that dynamical behaviour of the water molecules depends on the location of them in the crystal lattice. QE broadening is found to be temperature-dependent and increases on increase of temperature. The observed dynamics at low temperature corresponds to the non coordinated water molecules, and they undergo a localized translational diffusion. With increase of temperature, the coordinated ones become dynamic progressively. Data analysis showed that the dimension of the dynamical regime within which the water molecules undergo diffusion corresponds to the geometry of the structure of the Prussian blue compound.

\section{References}

[1] O. Kahn, Molecular Magnetism (VCH, New York, 1993)

[2] J. S. Miller and A. J. Epstein, Angew. Chem., Int. Ed. Engl. 33, 385 (1994)

[3] A. Kumar, S.M. Yusuf and L. Keller, Phys. Rev. B 71, 054414 (2005)

[4] P. Herren, P. Fischer, A. Ludi and W. Halg, Inorg. Chem. 19, 956 (1980)

[5] V. K. Sharma, S. Mitra, Amit Kumar, S. M. Yusuf, F. Juranyi and R. Mukhopadhyay, J. Phys.: Condens. Matter 23, 446002 (2011)

[6] V. K. Sharma, S. Mitra, Amit Kumar, S. M. Yusuf, F. Juranyi and R. Mukhopadhyay, AIP Conference Proceedings 1447, 949 (2012)

[7] A. J. Dianoux, Phil. Mag. B, 59, 17 (1989)

[8] V. Garcia Sakai, E. Mamontov, J. W. lynn, L. Viciu and R. J. Cava, Phys. Rev. B 75, 014505 (2007)

[9] F. Volino and A.J. Dianoux, Mol. Phys., 41, 271 (1980) 